\title{
A MATHEMATICAL MODEL FOR THE HARD SPHERE REPULSION IN IONIC SOLUTIONS*
}

\author{
YUNKYONG $\mathrm{HYON}^{\dagger}$, BOB EISENBERG ${ }^{\ddagger}$, AND CHUN LIU§
}

\begin{abstract}
We introduce a mathematical model for the finite size (repulsive) effects in ionic solutions. We first introduce an appropriate energy term into the total energy that represents the hard sphere repulsion of ions. The total energy then consists of the entropic energy, electrostatic potential energy, and the repulsive potential energy. The energetic variational approach derives a boundary value problem that includes contributions from the repulsive term with a no flux boundary condition for charge density which is a consequence of the variational approach, and physically implies charge conservation. The resulting system of partial differential equations is a modification of the Poisson-Nernst-Planck (PNP) equations widely if not universally used to describe the drift-diffusion of electrons and holes in semiconductors, and the movement of ions in solutions and protein channels. The modified PNP equations include the effects of the finite size of ions that are so important in the concentrated solutions near electrodes, active sites of enzymes, and selectivity filters of proteins. Finally, we do some numerical experiments using finite element methods, and present their results as a verification of the utility of the modified system.
\end{abstract}

Key words. Finite size effects, energetic variational approach, Poisson-Nernst-Planck equations, hard sphere, Lennard-Jones repulsive potential, hard sphere potential in density functional theory.

AMS subject classifications. 76A05, 76M99, 65C30.

\section{Introduction}

We introduce a mathematical model for ionic dynamics including the Brownian motion of ions, electrostatic interactions among charged ions, and finite size/volume (excluded volume) effects (steric effects) that uses a variational method to extend the classical literature $[17,21,41,42,43,44]$ from equilibrium to nonequilibrium. The finite size effects are an important part of a physical model of ions in water but have rarely been dealt with away from equilibrium, even though most applications of ion dynamics in fact involve ion flows.

Finite size effects do not occur in 'ideal' infinitely dilute solutions of independent components, but size effects are important in almost every other case where ionic solutions are involved [65]. Indeed, in most applications ions are concentrated in the special regions where they are important. For example, in electrochemistry ions are highly concentrated near electrodes. In biology ions are highly concentrated near the active sites of enzymes (the protein catalysts which do so much of life's chemistry), inside and near DNA and ion channels. In general, ions have their size, and their size is important: the dynamics of ions in small scale cannot be described without including finite size effects. A widely used model of an ion is a hard sphere of finite radius with charge located at the center. Much work has shown that a model of ionic solutions consisting of hard spheres in a uniform frictional dielectric does surprisingly well at equilibrium when all flows are zero. The literature of finite size effects is vast

*Received: June 10, 2010; accepted (in revised version): September 18, 2010. Communicated by Shi Jin.

${ }^{\dagger}$ Institute for Mathematics and Its Applications, University of Minnesota, 114 Lind, 207 Church St. S.E., Minneapolis, MN 55455, USA (hyon@ima.umn.edu).

${ }^{\ddagger}$ Department of Molecular Biophysics \& Physiology Rush Medical Center, 1653 West Congress, Parkway, Chicago, IL 60612, USA (beisenbe@rush.edu).

$\S$ Department of Mathematics, Pennsylvania State University, University Park, PA 16802, USA, and Institute for Mathematics and Its Applications, University of Minnesota, 114 Lind, 207 Church St. S.E., Minneapolis, MN 55455, USA (liu@math.psu.edu, or liu@ima.umn.edu). 
and includes many different treatments of finite size. Fortunately, the literature is well summarized in a recent book [65] and indeed in textbooks [42, 43]. We are not qualified to evaluate the different treatments of finite size effects because the issues are fundamentally those of physical chemistry, experimental and theoretical. But finite size effects are important. A leading worker writes "... it is still a fact that over the last decades, it was easier to fly to the moon than to describe the free energy of even the simplest salt solutions beyond a concentration of $0.1 \mathrm{M}$ or so." [65] Our contribution is to use a variational approach that extends the physics of previous equilibrium models to nonequilibrium [41, 42, 43, 44]. Previous extensions to nonequilibrium involving Density Functional Theory (of liquids) are discussed in Section 3.1.

Our research on the excluded volume of ions is particularly motivated by the study of the selectivity of ion channels in cell membranes $[15,16,19,20]$. Two key physical properties are the basis for the ion selectivity in these channels: one is electrostatic interactions with the side-chains of the protein that forms the channel; the other is the excluded volume effects of ions and side chains [17]. These physical properties can be included as parameters in a mathematical model of an individual ion. However, it is not so easy to obtain an appropriate mathematical system of equations for the flow of ions that includes such finite size effects $[58,59,60]$ and deals consistently with the electric field by satisfying sum rules $[57,66]$ and Gauss's law.

Our model in this paper can be directly linked to the selectivity phenomena of channels $[23,24,15,16]$. It can also be used to establish a time dependent model for ensembles of single channels $[22,24,27,28,25,26]$ that can be related to the classical Hodgkin-Huxley phenomenological representations. Although the excluded volume effect is one of the crucial determinants of the selectivity of channels, it is not the only one. A variety of other factors are involved including the properties of side chains of the channel protein (modified in mutation experiments), confinement in the small volume of the pore of the channel, the inhomogeneous dielectric coefficient of the channel protein, and channel pore and so on. Detailed modeling and numerical computations involving these features are presented elsewhere [18].

One of the advantages of the variational approach is that it deals directly and consistently with the coupling of the flow of ions one to another. The issues of coupling of ions one to another, and to the channel, have been central topics of biological investigation since the discovery of channels and transporters [67, 68, 69, 70, 71]. Indeed, channels and transporters were defined by the properties of the coupling between ion flows long before the proteins involved were isolated. Even today it is likely that structural measurements cannot actually resolve the functional difference between channels and transporters. The functional difference is the coupling of fluxes of ions. The variational approach deals directly with interactions of ions and coupling of fluxes.

In this paper, we concentrate on the specific subject of the finite size effects and its mathematical modeling. This has practical significance: for example, the only difference between the biologically crucial ions $\mathrm{Na}^{+}$and $\mathrm{K}^{+}$is their diameter; they are otherwise indistinguishable. We consider a hard sphere model for ions to verify the model. We use the energetic variational approach $[18,6,12]$ to determine the contribution of ion repulsion and finite size effects and create a modified PoissonNernst-Planck (PNP) model of ions in solution.

In the energetic variational approach we first define the total energy for the whole system of ions including hard sphere repulsion. The total energy consists of the entropic energy induced by the Brownian motion of ions, the electrostatic potential 
energy representing the coulomb interaction between the charged ions, and the repulsive potential energy caused by the excluded volume effect. (To avoid confusing some readers, we remark that we use the word 'energy' in the tradition of variational analysis. This is not the 'energy' of classical thermodynamics.) If we include only the entropy and electrostatic potential for the total energy, then the resulting system of partial differential equations (after variational derivatives are taken) is the classical PNP equation [6]. The PNP equations have been applied to solve charge transport problems in semiconductor and ion dynamics in biological phenomena $[13,14,8,45,46,47,48,49,50,51,61,62,63,64]$.

To perform our task, we need to define a proper repulsive potential energy describing the excluded volume energy in ion-ion interactions. There are several types of potential energy for the hard sphere repulsion: for instance, the Yukawa potential, the hard core potential, the repulsive term of Lennard-Jones (LJ) potential, and the hard sphere potential in Rosenfeld's [35, 36, 52, 53, 54] density functional theory (DFT) of fluids [55, 56, 57, 38], which is different from the DFT of electrons used in Quantum Chemistry. In this work, we employ the LJ repulsive potential for the excluded volume effects [11] and also the hard sphere potential in DFT [29, 30, 35, 36] for comparison. Then we take variational derivatives of the total energy for the case of the LJ repulsive potential and the hard sphere chemical potential of DFT. This leads us to a system of equations including the contributions of the finite size effect that we call the modified PNP system. These equations are of course different for the hard sphere and DFT descriptions of finite size effects.

We also demonstrate the utility of this description of finite size effects by showing some numerical computations for the modified PNP system. Since the energetic variational approach is based on the variational structure of the underlying mathematical model, it is very important to preserve the characteristic variational structure in computational methods as well as analysis. For that reason, we use finite element methods in numerical computations of the modified PNP system previously shown to preserve the important characteristics of the variational procedure $[6,12]$.

The organization of the paper is this: In the next section, we briefly discuss the PNP equations and summarize their derivation through the energetic variational approach. Section 3 covers the derivation of the modified PNP that includes the finite size effect after defining a proper total energy. In Section 4, we present several numerical experiments for the verification of the modified PNP equations, and especially the finite size effects. Finally, we make some general remarks, looking to the future.

\section{Poisson-Nernst-Planck equations}

The PNP equations for electrokinetic theory are

$$
\begin{aligned}
\frac{\partial c_{i}}{\partial t} & =\nabla \cdot\left(D_{i}\left(\nabla c_{i}+\frac{z_{i} e}{k_{B} T} c_{i} \nabla \phi\right)\right), \\
\nabla \cdot(\varepsilon \nabla \phi) & =-\left(\rho_{0}+\sum_{i=1}^{N} z_{i} e c_{i}\right)
\end{aligned}
$$

where $c_{i}$ is the ion density for $i$ th species, $D_{i}$ is the diffusion constant, $z_{i}$ is the valence, $e$ is the unit charge, $k_{B}$ is the Boltzmann constant, $T$ is the absolute temperature, $\varepsilon$ is the dielectric constant, $\phi$ is the electrostatic potential, $\rho_{0}$ is the permanent (fixed) charge density of the system, and $N$ is the number of ions. 
The system (2.1), (2.2) satisfies the following dissipative energy law:

$$
\begin{aligned}
& \frac{d}{d t} \int\left\{k_{B} T \sum_{i=1}^{N} c_{i} \log c_{i}+\frac{1}{2}\left(\rho_{0}+\sum_{i=1}^{N} z_{i} e c_{i}\right) \phi\right\} d \vec{x} \\
= & -\int\left\{\sum_{i=1}^{N} \frac{D_{i} c_{i}}{k_{B} T}\left|k_{B} T \frac{\nabla c_{i}}{c_{i}}+z_{i} e \nabla \phi\right|^{2}\right\} d \vec{x} .
\end{aligned}
$$

The second term in the total energy, the left hand side of (2.3), can be rewritten as $\varepsilon \frac{|\nabla \phi|^{2}}{2}$ under the proper boundary condition for electrostatic potential. This is the electrostatic potential energy with the definition of electric field, $\vec{E}=-\nabla \phi$, in classical electromagnetic theory [1].

When an energy equation is well defined to describe a physical phenomenon, one can derive a system of differential equations satisfying the energy equation by the energetic variational approach. In other words, the energetic variational approach forms a framework (or procedure) that derives a system of equations corresponding to the energy law. Thus, for the PNP system (2.1), (2.2), one can derive (2.1) from (2.3) using the energetic variational approach [6].

The derivation of PNP system from the energy is not a trivial task. The variational calculus needs additional ingredients. In the energetic variational approaches, the variational derivative, $\frac{\delta E^{\text {total }}}{\delta c_{i}}$, gives the chemical potential $\mu_{i}$ for each $i$ th ion, $i=1, \cdots, N$. The Nernst-Planck equation (2.1) is then given by

$$
\frac{\partial c_{i}}{\partial t}=\nabla \cdot\left(\frac{D_{i}}{k_{B} T} c_{i} \nabla \mu_{i}\right), \quad \text { for } i=1, \cdots, N
$$

To make this paper self-contained, we briefly summarize the derivation of the NernstPlanck equation (2.1) from the energy (2.3). For the derivation we note the electrostatic potential $\phi$ with the Green's kernel $G(\vec{x}, \vec{y})$ is

$$
\phi(\vec{x})=-\frac{4 \pi}{\varepsilon} \int G(\vec{x}, \vec{y})\left(\rho_{0}+\sum_{i=1}^{N} z_{i} e c_{i}\right) d \vec{y} .
$$

The total energy $E$ which is in the left-hand side of (2.3) is

$$
E^{\text {total }}=\int\left\{k_{B} T \sum_{i=1}^{N} c_{i} \log c_{i}+\frac{1}{2}\left(\rho_{0}+\sum_{i=1}^{N} z_{i} e c_{i}\right) \phi\right\} d \vec{x} .
$$

We can easily see that the electrostatic potential is not independent of the ion densities $c_{i}$, which is physically obvious because the densities describe charge. Hence when we take the variational derivative with respect to $c_{i}$ we need to consider the electrostatic potential as a functional of the ion density. Thus, we have that

$$
\begin{gathered}
\delta E=\int\left[k_{B} T\left(\log c_{i}+1\right) \delta c_{i}+\frac{1}{2}\left\{\frac{4 \pi z_{i} e \delta c_{i}}{\varepsilon} \int G(\vec{x}, \vec{y})\left(\rho_{0}+\sum_{i=1}^{N} z_{i} e c_{i}(\vec{y})\right) d \vec{y}\right.\right. \\
\left.\left.+\left(\rho_{0}+\sum_{i=1}^{N} z_{i} e c_{i}\right) \frac{4 \pi z_{i} e}{\varepsilon} \int G(\vec{x}, \vec{y}) \delta c_{i} d \vec{y}\right\}\right] d \vec{x}, \quad \text { for } i=1, \cdots, N .
\end{gathered}
$$


Combining the last two terms in (2.7), we obtain

$$
\delta E=\int\left\{k_{B} T\left(\log c_{i}+1\right)+z_{i} e \phi\right\} \delta c_{i} d \vec{x}, \quad \text { for } i=1, \cdots, N .
$$

Hence we obtain the chemical potential $\mu_{i}$ :

$$
\mu_{i}=k_{B} T\left(\log c_{i}+1\right)+z_{i} e \phi, \quad \text { for } i=1, \cdots, N .
$$

Therefore, using (2.4) we obtain the Nernst-Planck equation,

$$
\begin{aligned}
\frac{\partial c_{i}}{\partial t} & =\nabla \cdot\left(\frac{D_{i}}{k_{B} T} c_{i} \nabla\left(k_{B} T\left(\log c_{i}+1\right)+z_{i} e \phi\right)\right) \\
& =\nabla \cdot\left(D_{i}\left(\nabla c_{i}+\frac{z_{i} e}{k_{B} T} c_{i} \nabla \phi\right)\right) .
\end{aligned}
$$

A simplification leads us to the Nernst-Planck equation (2.1) for the ion density $c_{i}$, for $i=1, \cdots, N$.

The framework of the energetic variational approach is briefly summarized as follows:

i. Find a mathematical description of the physical phenomenon of interest.

ii. Define an appropriate energy equation to describe the desired physical phenomenon.

iii. Apply the energetic variational approach to obtain a system of differential equations.

iv. Verify the system of equations in numerical experiments, and mathematical analysis.

REMARK 2.1. Note that the boundary condition for charge density is a direct consequence of the energy variational principle. In this sense, the natural boundary condition for charge density is a no flux boundary condition that itself - without additional assumptions - implies charge conservation of the system. One may not explicitly see the point in the derivation of the chemical potential $\mu_{i}$ from (2.6) to (2.10), but one can easily see the consequences of the no flux boundary condition by looking at our derivation of the energy dissipation law (2.6) 1rom the PNP equation (2.10).

\section{Modified PNP system with hard sphere repulsion}

In this section we discuss the excluded volume effects in a hard sphere model of ionic fluids. In chemical physics of fluids on the atomic scale and in colloidal suspensions, the excluded volume effect is a crucial physical factor for modeling hard sphere mixtures [31]. The excluded volume often appears in physical chemistry in the equation of state (EOS); for instance, a well-known EOS is the Van der Waals equation.

The frontier in this measurement is the Carnahan-Starling EOS [32] which is obtained as a solution of the Percus-Yevick (PY) equation [33] for hard sphere mixtures. From the Carnahan-Starling EOS, there are many extensions for more accurate measurement (references are in [37]). Related to the hard sphere mixtures, there is a well-known approach, called density functional theory (DFT) of fluids $[35,36,52,53,54,55,56,57,38]$, which differs from the DFT of electrons in quantum chemistry. In the following subsection, we briefly discuss DFT. 
In Subsection 3.2 we utilize the excluded volume energy, which is a nonlocal type of repulsion potential proposed in [11]. Then we define the total energy with the repulsive potential energy, and derive a modified PNP system including the hard sphere repulsion. The detailed derivation is presented in Subsection 3.2.

3.1. Density functional theory. The DFT is based on the fundamental measure theory for uncharged (atomic) fluids [35, 36]. In Rosenfeld's DFT the energy density function is introduced for the free energy functional including the hard sphere repulsion energy. Moreover, DFT uses an (underived) ansatz for the energy density function. From the energy density function one can separately obtain the chemical potentials for hard sphere and electrostatic interactions. The errors in this ansatz are unknown and customarily estimated by comparison with Monte Carlo simulations of hard spheres.

PNP and DFT were coupled in a straightforward approach in [39], and the model called PNP-DFT shows significant results for ionic mixtures, especially the layering phenomena of charge density near a charged wall [39]. However, it is not clear that PNP-DFT satisfies the sum rules of statistical mechanics $[57,66]$ or Gauss' law of electrostatics and it does not allow changes in the shape of the ionic atmosphere with flow, as discussed in $[58,59,60]$.

However, the approach leaves out many effects (e.g., electrophoretic and relaxation terms) known to be important in determining the conductance of ionic solutions $[58,59,60]$. These terms are probably small enough to be neglected when ions flow through a protein channel, judging from the success of the model that leaves them out [40]. That success cannot be guaranteed by the derivation of the PNP-DFT theory because these terms are quite important in other cases, and it is in fact not safe to assume they are uniformly small in any situation, including ion channels.

Here, we derive the chemical potential of hard sphere repulsion from the interpretation of the energetic variational framework using results from Rosenfeld's DFT. First, let $\Phi_{H S}$ be the energy functional for the hard sphere. Then the energy of the uncharged hard spheres is defined in this DFT by

$$
E_{H S}^{D F T}=\int \Phi_{H S}\left(\left\{n_{\alpha}\right\},\left\{\vec{n}_{\beta}\right\}\right) d \vec{x}, \quad \text { for } \alpha=0, \cdots, 3, \beta=4,5
$$

where

$$
\begin{gathered}
\Phi_{H S}\left(\left\{n_{\alpha}\right\},\left\{\vec{n}_{\beta}\right\}\right)=-n_{0} \log \left(1-n_{3}\right)+\frac{n_{1} n_{2}-\vec{n}_{4} \cdot \vec{n}_{5}}{1-n_{3}} \\
+\frac{n_{2}^{3}}{24 \pi\left(1-n_{3}\right)^{2}}\left(1-\frac{\left|\vec{n}_{5}\right|^{2}}{n_{2}^{2}}\right)^{3}, \\
n_{\alpha}=\sum_{i}^{N} \int c_{i}(\vec{y}) \omega_{i}^{(\alpha)}(\vec{y}-\vec{x}) d \vec{y}, \quad \text { for } \quad \alpha=0, \cdots, 3, \\
\vec{n}_{\beta}=\sum_{i}^{N} \int c_{i}(\vec{y}) \vec{\omega}_{i}^{(\beta)}(\vec{y}-\vec{x}) d \vec{y}, \quad \text { for } \beta=4,5 .
\end{gathered}
$$


The weight functions $\omega_{i}^{(\alpha)}, \vec{\omega}_{i}^{(\beta)}$, for $i=1, \cdots, N, \alpha=0, \cdots, 3, \beta=4,5$ are given by

$$
\begin{aligned}
4 \pi a_{i}^{2} \omega_{i}^{(0)}(\vec{r}) & =4 \pi a_{i} \omega_{i}^{(1)}(\vec{r})=\omega_{i}^{(2)}(\vec{r}), \\
4 \pi a_{i} \vec{\omega}_{i}^{(4)}(\vec{r}) & =\vec{\omega}_{i}^{(5)}(\vec{r}), \\
\omega_{i}^{(2)}(\vec{r}) & =\delta\left(|\vec{r}|-a_{i}\right), \\
\omega_{i}^{(3)}(\vec{r}) & =\theta\left(|\vec{r}|-a_{i}\right), \\
\vec{\omega}_{i}^{(5)}(\vec{r}) & =\frac{\vec{r}}{|\vec{r}|} \delta\left(|\vec{r}|-a_{i}\right)
\end{aligned}
$$

where $a_{i}$, for $i=1, \cdots, N$ are the radius of ion species $i, \delta(r)$ is the Dirac delta function, and $\theta(r)$ is the unit step function defined as

$$
\theta(r)= \begin{cases}0, & r \geq 0, \\ 1, & r<0 .\end{cases}
$$

Through the variational derivative, $\frac{\delta E_{H S}^{D F T}}{\delta c_{i}}$ gives us the chemical potential $\mu_{i}^{H S}$, for $i=1, \cdots, N$,

$$
\begin{aligned}
\mu_{i}^{H S}=k_{B} T & \left(\sum_{\alpha=0}^{3} \int \frac{\partial \Phi_{H S}}{\partial n_{\alpha}}(\vec{y}) \omega_{i}^{(\alpha)}(\vec{x}-\vec{y}) d \vec{y}\right. \\
& \left.+\sum_{\beta=4}^{5} \int \frac{\partial \Phi_{H S}}{\partial \vec{n}_{\beta}}(\vec{y}) \vec{\omega}_{i}^{(\beta)}(\vec{x}-\vec{y}) d \vec{y}\right) .
\end{aligned}
$$

If we impose this hard sphere chemical potential $\mu_{i}^{H S}$ into the Nernst-Planck equation (2.4), then we have a modification of the Nernst-Planck equation,

$$
\frac{\partial c_{i}}{\partial t}=\nabla\left(\frac{D_{i}}{k_{B} T} c_{i} \nabla\left(\mu_{i}^{P N P}+\mu_{i}^{H S}\right)\right), \quad \text { for } i=1, \cdots, N
$$

where $\mu_{i}^{P N P}$, for $i=1, \cdots, N$ is the chemical potential $\mu_{i}$ in (2.4).

The coupling with the Poisson equation (2.2) gives us a modified PNP system with DFT hard sphere repulsion potential. Moreover, this modified system satisfies the following energy equation:

$$
\begin{aligned}
& \frac{d}{d t} \int\left\{k_{B} T \sum_{i=1}^{N} c_{i} \log c_{i}+\frac{1}{2}\left(\rho_{0}+\sum_{i=1}^{N} z_{i} e c_{i}\right) \phi+\Phi_{H S}\left(\left\{n_{\alpha}\right\},\left\{\vec{n}_{\beta}\right\}\right)\right\} d \vec{x} \\
= & -\int\left\{\sum_{i=1}^{N} \frac{D_{i} c_{i}}{k_{B} T}\left|k_{B} T \frac{\nabla c_{i}}{c_{i}}+z_{i} e \nabla \phi+\nabla \mu_{i}^{H S}\right|^{2}\right\} d \vec{x} .
\end{aligned}
$$

3.2. Lennard-Jones hard sphere repulsion. In this subsection we use the energetic variational approach to derive a system of differential equations including hard sphere repulsion using the LJ repulsive potential. To include the repulsive effect of ions which are modeled as hard spheres, we first define an appropriate energy term.

In ion-ion interaction, a regularized repulsive interaction potential is introduced in $[5]$ as

$$
\Psi_{i, j}(|\vec{x}-\vec{y}|)=\frac{\varepsilon_{i, j}\left(a_{i}+a_{j}\right)^{12}}{|\vec{x}-\vec{y}|^{12}}
$$


for $i$ th and $j$ th ions located at $\vec{x}$ and $\vec{y}$ with the radii $a_{i}, a_{j}$, respectively, where $\varepsilon_{i, j}$ is an appropriate energy constant, chosen empirically. Then the contribution of the repulsive potential $\Psi$ to the total free energy is defined by

$$
E_{i, j}^{r}=\frac{1}{2} \iint \Psi_{i, j}(|\vec{x}-\vec{y}|) c_{i}(\vec{x}) c_{j}(\vec{y}) d \vec{x} d \vec{y}
$$

where $c_{i}, c_{j}$ are the densities of $i$ th, $j$ th ions, respectively.

For the sake of simplicity in this derivation, we consider a two-ion system with the charge densities $c_{n}, c_{p}$. All derivations and programs have been written for a multiple ion system, with ions of any charge. Then, the total repulsive energy is defined by

$$
E^{r}=\sum_{i=n, p} \sum_{j \neq i} E_{i, j}^{r}=\sum_{i=n, p} \sum_{j \neq i} \frac{1}{2} \iint \Psi_{i, j}(|\vec{x}-\vec{y}|) c_{i}(\vec{x}) c_{j}(\vec{y}) d \vec{x} d \vec{y} .
$$

We here take a variational derivative with respect to each ion, $\frac{\delta E^{r}}{\delta c_{i}}$ to obtain the repulsive energy term into the system of equations. This leads us to the following Nernst-Planck equations for the charge densities, $c_{n}, c_{p}$ :

$$
\begin{aligned}
\frac{\partial c_{n}}{\partial t}=\nabla & \cdot\left[D _ { n } \left\{\nabla c_{n}+\frac{c_{n}}{k_{B} T}\left(z_{n} e \nabla \phi-\int \frac{12 \varepsilon_{n, n}\left(a_{n}+a_{n}\right)^{12}(\vec{x}-\vec{y})}{|\vec{x}-\vec{y}|^{14}} c_{n}(\vec{y}) d \vec{y}\right.\right.\right. \\
& \left.\left.\left.-\int \frac{6 \varepsilon_{n, p}\left(a_{n}+a_{p}\right)^{12}(\vec{x}-\vec{y})}{|\vec{x}-\vec{y}|^{14}} c_{p}(\vec{y}) d \vec{y}\right)\right\}\right], \\
\frac{\partial c_{p}}{\partial t}=\nabla & \cdot\left[D _ { p } \left\{\nabla c_{p}+\frac{c_{p}}{k_{B} T}\left(z_{p} e \nabla \phi-\int \frac{12 \varepsilon_{p, p}\left(a_{p}+a_{p}\right)^{12}(\vec{x}-\vec{y})}{|\vec{x}-\vec{y}|^{14}} c_{p}(\vec{y}) d \vec{y}\right.\right.\right. \\
& \left.\left.\left.-\int \frac{6 \varepsilon_{n, p}\left(a_{n}+a_{p}\right)^{12}(\vec{x}-\vec{y})}{|\vec{x}-\vec{y}|^{14}} c_{n}(\vec{y}) d \vec{y}\right)\right\}\right] .
\end{aligned}
$$

The details of the derivation of the repulsive terms in the chemical potentials are presented in Appendix A. We now have the coupled system (2.2), (3.17), (3.18) including finite size effects. We will call the system a modified PNP system. One advantage of the variational approach is the fact that the resulting system, the modified PNP, naturally satisfies the energy dissipation law,

$$
\begin{aligned}
& \frac{d}{d t} \int\left\{k_{B} T \sum_{i=n, p} c_{i} \log c_{i}+\frac{1}{2}\left(\rho_{0}+\sum_{i=n, p} z_{i} e c_{i}\right) \phi+\sum_{i, j=n, p} \frac{c_{i}}{2} \int \Psi_{i, j} c_{j} d \vec{y}\right\} d \vec{x} \\
= & -\int\left\{\sum_{i=n, p} \frac{D_{i} c_{i}}{k_{B} T}\left|k_{B} T \frac{\nabla c_{i}}{c_{i}}+z_{i} e \nabla \phi-\sum_{j=n, p} \nabla \int \tilde{\Psi}_{i, j} c_{j} d \vec{y}\right|^{2}\right\} d \vec{x}
\end{aligned}
$$

where $\tilde{\Psi}_{i, j}=12 \Psi_{i, j}$ for $i=j$, and $\tilde{\Psi}_{i, j}=6 \Psi_{i, j}$ for $i \neq j$.

\section{Numerical simulations}

In this section we present some numerical results as a verification of the finite size effects with the modified PNP equations, (2.2), (3.17), (3.18). We consider 1dimensional domains with two opposite monovalent ions, i.e., $z_{n}=-1, z_{p}=1$ and the same radii, $a_{n}=a_{p}=1.5 \AA$. Throughout the computations we use no-flux boundary 
conditions for the ion concentrations. The boundary conditions for the electrostatic potential will be specified in the following discussions.

In DFT, the Rosenfeld functional has been developed from a 1-dimensional study of inhomogeneous hard sphere mixture [29, 30]. The reduction of DFT to 1-dimensional space is given as follows:

$$
E_{H S}^{D F T_{1 d}}=\int \Phi_{H S_{1 d}}\left(\left\{n_{\alpha}\right\}\right) d x \quad \text { for } \alpha=0,1
$$

where

$$
\begin{aligned}
n_{\alpha}(x) & =\sum_{i=1}^{N} \int c_{i}(y) \omega_{i}^{(\alpha)}(x-y) d y, \\
\omega_{i}^{(0)}(z) & =\frac{1}{2}\left\{\delta\left(z-a_{i}\right)+\delta\left(z+a_{i}\right)\right\}, \\
\omega_{i}^{(1)}(z) & =\theta\left(|z|-a_{i}\right) .
\end{aligned}
$$

Then the chemical potential for the hard spheres is given by

$$
\begin{aligned}
\mu_{i}^{H S_{1 d}} & =k_{B} T \sum_{\alpha=0}^{1} \int \frac{\partial \Phi}{\partial n_{\alpha}}(y) \omega_{i}^{(\alpha)}(x-y) d y \\
& =k_{B} T \int\left\{-\log \left(1-n_{1}\right) w_{i}^{(0)}(x-y)+\frac{n_{0}}{1-n_{1}} \omega_{i}^{(1)}(x-y)\right\} d y
\end{aligned}
$$

Then we substitute the hard sphere repulsion potential $\mu_{i}^{H S}$ (3.11) with $\mu_{i}^{H S_{1 d}}$ in (4.5) for the $i$ th ion species.

To solve the system of equations we use finite element methods, especially the edge averaged finite element method (EAFE) which is developed for drift-diffusion type of equation in [7], to solve the modified Nernst-Planck equation (3.17), (3.18). A standard finite element method is used for the electrostatic potential $[3,4]$. To ensure self consistency between ionic concentrations and the electrostatic potential solutions we employ a convex iteration scheme $[2,6]$. The iterative algorithm to solve the modified PNP system is summarized in the following.

Remark 4.1. Note that Algorithm 4.1 does not satisfy the finite dimensional (numerical) energy law. The variational approach for the energy law helps us to find the analytic system of equations. However, it requires a specific variational structure corresponding to the physical structure of the problem. In the case of modified PNP equations, even PNP, the appropriate finite dimensional (numerical) realization of such an energy law is a totally open question. The specific variational structure of the modified PNP equations, and even of PNP itself, requires an $L^{1} \log L^{1}$ space for the energy law where $L^{1}$ is the $L^{p}$ space in mathematics when $p=1$. But the appropriate numerical implementation of that in a finite dimensional space is unknown. There are some attempts to deal with this issue in [73], but it is still quite open. Our future work on the modified PNP equations needs to deal with this issue, among others.

In numerical computations of the modified PNP system, one obstacle is the nonlocal repulsive term in integral form. It is expensive in computational time and is hard to compute accurately. We use a backward Euler method in the time variable to deal with the ion concentration variables $c_{n}, c_{p}$. We use a semi-implicit type 


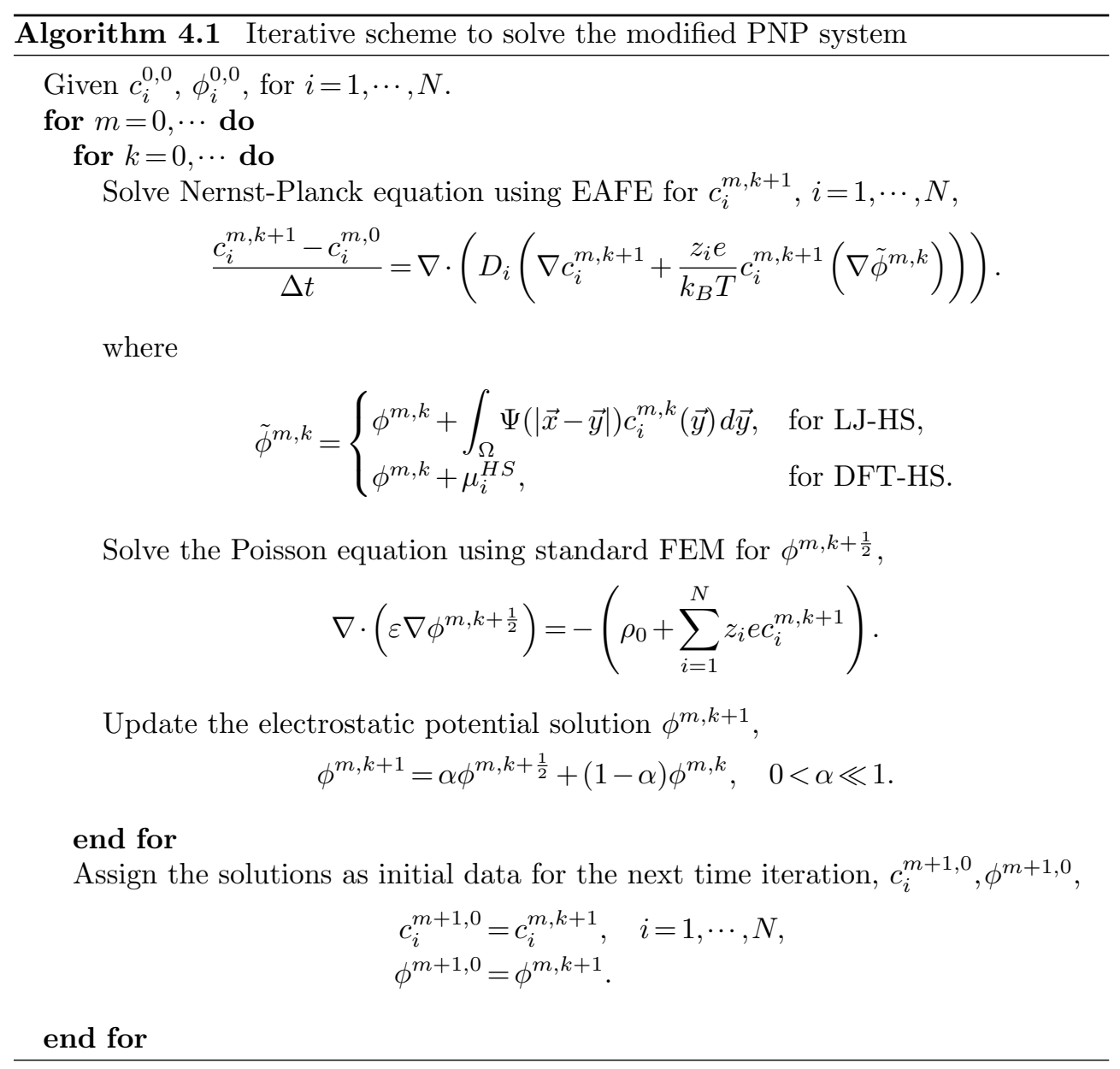

of self-consistent (inner) iteration between ion concentrations and the electrostatic potential. In non-local repulsion terms we use the previous step value for the ion concentration. In this case, we have to be careful to have a small enough time step to ensure convergence of the numerical scheme.

The LJ repulsive kernel is intrinsically singular when ions are overlapped, i.e., $\vec{x}=\vec{y}$. For that reason, a separate treatment of this singular behavior is required in numerical computations. We apply a cut-off in integral domain with respect to the size of ions instead of the integration over the whole domain. An obvious choice of cut-off is

$$
\int_{|\vec{x}-\vec{y}| \geq R_{n, n}} \frac{12 \varepsilon_{n, n}\left(a_{n}+a_{n}\right)^{12}(\vec{x}-\vec{y})}{|\vec{x}-\vec{y}|^{14}} c_{n}(\vec{y}) d \vec{y}
$$

for the first repulsion term in (3.17) where $R_{i, j}=a_{i}+a_{j}$ for $i, j=n, p$. Choosing a cutoff is very sensitive in computations, and is automatically connected to the stability. When a large value for $R_{i, j}$ is chosen, the contribution of finite size could be lost. On the other hand, if a small value is chosen, then numerical instability may occur.

REMARK 4.2. The cut-off of the nonlocal repulsive term (4.6) used in numerical calculations can be a smaller value of $R_{i, j}$ than $R_{i, j}=a_{i}+a_{j}$. The choice of cut- 
off is related to the strength of repulsion potential. The repulsive kernel $\Psi_{i, j}$ can be chosen in a different form, which approximates experimental data and is related to the hardness/softness of ions.

The time consumed in evaluating the non-local repulsion terms is a significant limitation. A fast Fourier transformation (FFT) might allow a different and local representation of the repulsion term that allows faster computation and is accurate enough. The local representation would be best determined by a systematic approximation procedure based on the fundamental properties used in the original derivation of the nonlocal repulsive terms used in this paper.
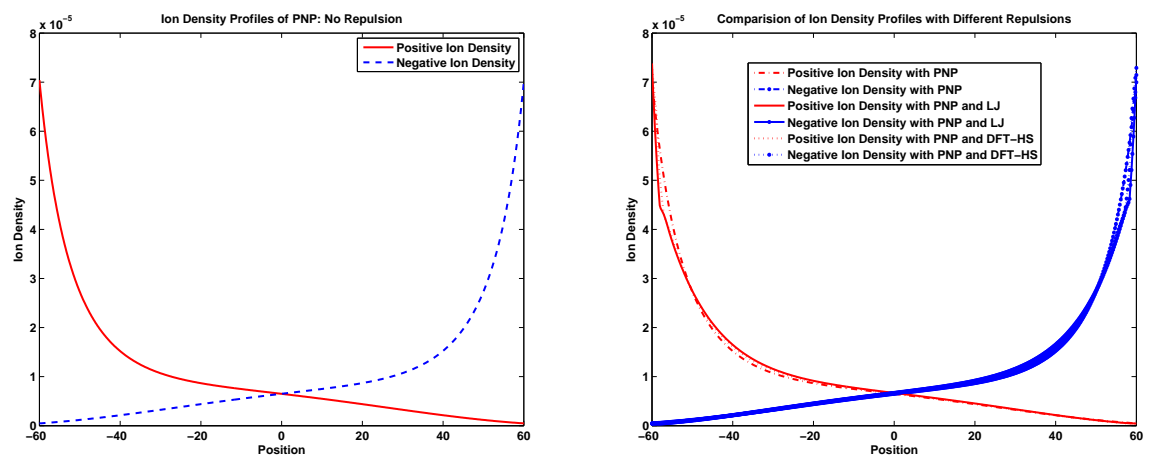

FIG. 4.1. The ionic concentration profile without finite size effects (left), and the comparison of ionic concentrations with the finite size effects, LJ repulsive potential and DFT hard sphere potential (right) under the Robin boundary condition.
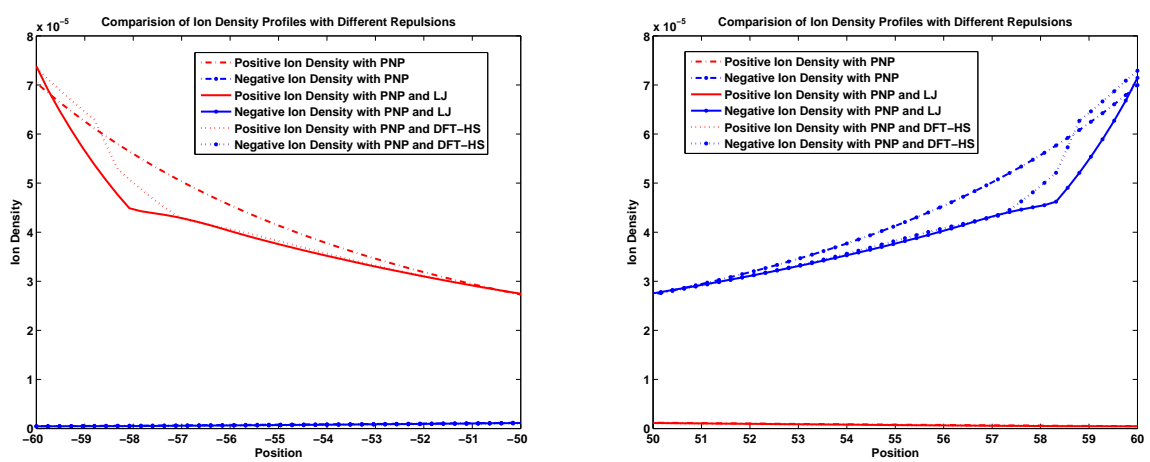

FIG. 4.2. The zoom-in pictures of the left-hand side picture in Figure 4.1 near boundary.

So that our numerical computations observe the finite size effects, we consider the zero permanent charge density case in the system, that is, $\rho_{0}=0$ in (2.2). The computational domain is $\Omega=[-60,60]$. The Robin boundary conditions for the electrostatic potential are chosen so that

$$
\left.\left(\phi-\eta \frac{\partial \phi}{\partial x}\right)\right|_{x=-60}=\left.\left(\phi+\eta \frac{\partial \phi}{\partial x}\right)\right|_{x=60}=0
$$


Throughout the numerical experiments we use $\eta=\left(\varepsilon_{r} \varepsilon_{0}\right)^{4}$, where $\varepsilon_{r}$ is the relative permittivity, 78.5 for water, and $\varepsilon_{0}$ is the permittivity of vacuum, $8.8542 \frac{\mathrm{C}}{\mathrm{Vm}}$. This simple situation shows a finite size effect. We compare modified and pure PNP equations with the same computational condition except the repulsion term. The comparison of these results is presented in the right-hand side panel of Figure 4.1. The left-hand side panel in Figure 4.1 is for the ion density profiles of PNP system without any finite size effects for clarification. According to the results, both numerical solutions to PNP with LJ repulsive potential and with DFT hard sphere potential have the same overall behavior of ion concentration, but in detail the ion concentrations show a different profile. In particular, the difference near the boundary is larger than that in bulk. In this case, the largest contribution of the repulsion term is apparent near the boundary. It is caused by the high concentration of ions near the boundary obeying the electrostatic field. The zoom-in panels in Figure 4.2 show the behavior of the solutions in more detail. One can easily observe that the DFT hard sphere potential gives a more complex behavior than the LJ repulsive potential. The differences between LJ and DFT clearly deserve investigation but we believe that this is not very useful unless both calculations, as well as Monte Carlo simulations, are done in three dimensions. We are working hard on this.

REMARK 4.3. In Figure 4.2 the discontinuous behavior of the gradient of charge density near the wall is obvious. One-dimensional DFT computations also show this behavior [30] and it is in fact well known in the literature of one dimensional excluded volume effects; see references in [72].

Next, we consider a little more complicated situation that has a charged wall. The right-hand side boundary/wall is negatively charged. The left-hand side boundary/wall has no charge. We establish the charged wall through the variable $\rho_{0}$ in the Poisson equation (2.2). We set $\rho_{0}=0$ on the left-hand side boundary, and $\rho_{0}=1$ on the right-hand side boundary. The domain $\Omega$ is $[-10,10]$. In this case, we impose the Dirichlet and Neumann boundary conditions for the left-hand side and the right-hand side boundary, respectively:

$$
\left.\phi\right|_{x=-10}=0,\left.\quad \frac{\partial \phi}{\partial \vec{\nu}}\right|_{x=10}=0
$$

where $\vec{\nu}$ is the unit outer normal vector.

The numerical results with one charged wall is presented in Figure 4.3. The lefthand side panel in Figure 4.3 is the ion concentration profiles without any finite size effects and the right-hand side panel is those with the finite size effects. The results on the right-hand side panel shows the very important phenomena of the finite size effects - the so called layering phenomena (charge inversion). The comparison shows a rigorous evidence of the finite size effects in the modified PNP system.

For more detailed comparison, we present the zoom-in pictures near the boundaries in Figure 4.4. One can easily see the contribution of the finite size effects and the difference between the PNP and the modified PNP equations. The DFT hard sphere potential gives more complex behavior than the LJ repulsive potential. Complex behavior is particularly visible in Figures 4.2 and 4.4, which seems to show a derivative discontinuity near the boundary (around position \pm 9.85 ). Behavior of this complexity is seen in previous computations of similar one dimensional geometry [30] and is illustrated in [72]. 

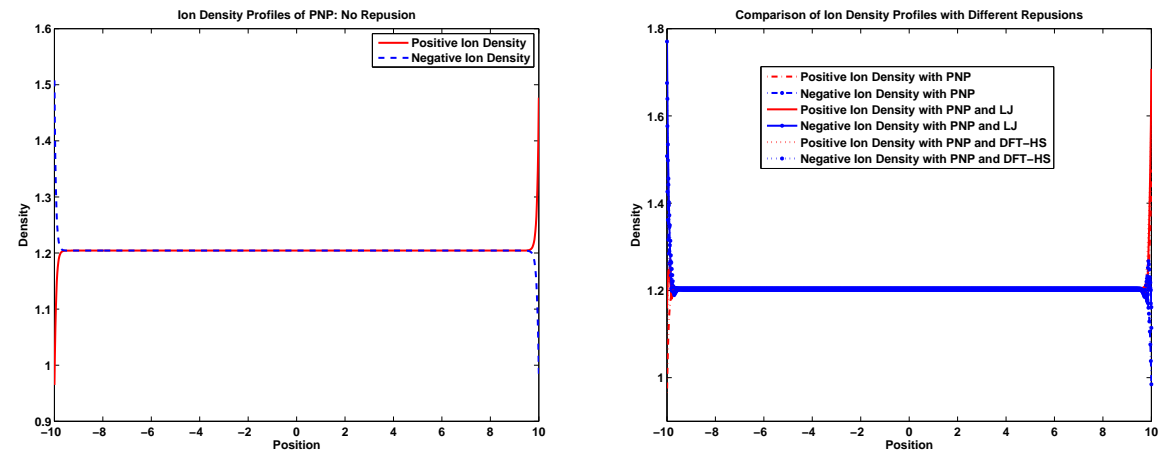

FIG. 4.3. The ionic concentration profile without finite size effects (left), and the comparison of ionic concentrations with the finite size effects, LJ repulsive potential and DFT hard sphere potential (right) under the no-flux boundary condition.
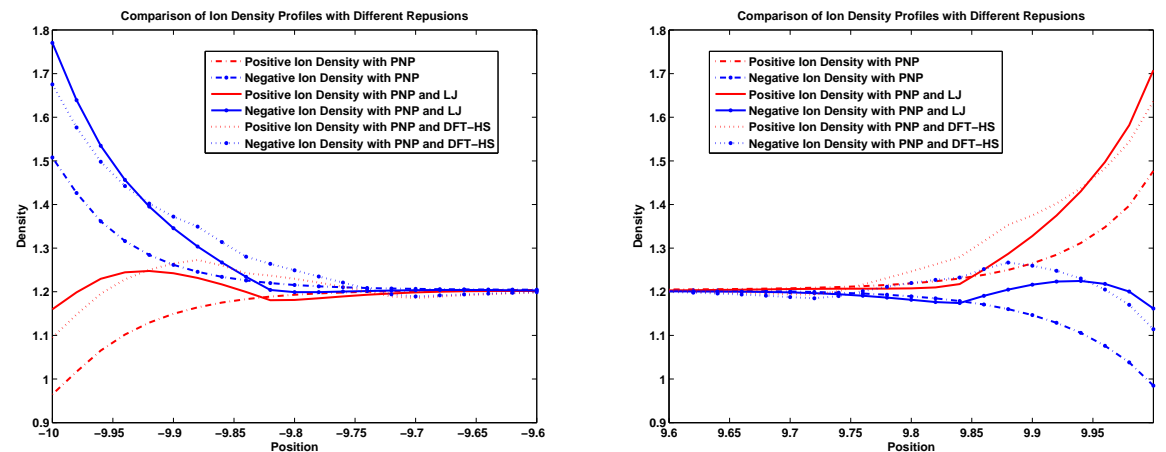

Fig. 4.4. The zoom-in pictures of the left-hand side picture in Figure 4.3 near boundary

\section{Conclusion}

The purpose of this present paper is to develop a mathematical model for the finite size effect using a regularized LJ repulsive potential under the energy variational framework, and its numerical verification to recover a layering behavior which is a wellknow feature of finite size effects. We have introduced a mathematical model system, modified PNP, for ionic solutions including hard sphere repulsion. The modified PNP has been derived from the energy dissipation law using the energetic variational approach. We also presented some numerical results showing the finite size effects in modified PNP equations.

Looking to the future, we note that our numerical schemes do not guarantee convergence for high ion concentration cases. In this case an improved numerical scheme is required, such as adaptive mesh refinement near the boundary, to catch the boundary layers. Developing a numerical method which preserves the finite dimensional dissipative energy law is another task important for the practical application of these models. We will further analyze the mathematical structure of the modified PNP equations in other papers. We will report additional numerical methods there and 
elsewhere.

The model and calculations might be improved by using another type of repulsive potential instead of the Lennard-Jones or DFT hard sphere repulsion potentials we use here. Moreover, the comparison of PNP equations with different types of repulsive potentials would also reveal interesting physics and biophysics. The electrodiffusion of finite sized spheres underlies an enormous range of phenomena central to biology, chemistry, and physics. Indeed, the crucial ions $\mathrm{Na}^{+}$and $\mathrm{K}^{+}$differ only because they are charged and have different diameters. Ion solutions are not made of uncharged point particles and it is necessary for mathematics to deal with that reality; investigation of the mathematical properties of PNP with finite size effects is necessary. It is also likely to be useful and interesting.

Acknowledgement. We would like to thank Professor Wolfgang Nonner, Dezső Boda, Dirk Gillespie, and Roland Roth for a communication on the DFT related to the hard sphere repulsion. Chun Liu is supported by NSF grant DMS-0707594. Bob Eisenberg was supported in part by NIH grant GM076013.

Appendix A. The variational derivative of the total repulsive energy. We here present the detailed derivation of the finite size effect terms in (3.17)-(3.18).

$$
\begin{aligned}
\delta E^{\text {repulsion }}=\frac{1}{2} \iint & \frac{\varepsilon_{n, n}\left(a_{n}+a_{n}\right)^{12}}{|\vec{x}-\vec{y}|^{12}} \delta c_{n}(\vec{x}) c_{n}(\vec{y}) d \vec{x} d \vec{y} \\
& +\frac{1}{2} \iint \frac{\varepsilon_{n, n}\left(a_{n}+a_{n}\right)^{12}}{|\vec{x}-\vec{y}|^{12}} c_{n}(\vec{x}) \delta c_{n}(\vec{y}) d \vec{x} d \vec{y} \\
& +\frac{1}{2} \iint \frac{\varepsilon_{n, p}\left(a_{n}+a_{p}\right)^{12}}{|\vec{x}-\vec{y}|^{12}} \delta c_{n}(\vec{x}) c_{p}(\vec{y}) d \vec{x} d \vec{y}
\end{aligned}
$$

Therefore, we have the repulsive term $\mu_{c_{n}}^{r}$, in the chemical potential for NernstPlanck equation, of the charge density $c_{n}$ :

$$
\mu_{c_{n}}^{r}=\int \frac{12 \varepsilon_{n, n}\left(a_{n}+a_{n}\right)^{12}}{|\vec{x}-\vec{y}|^{12}} c_{n}(\vec{y})+\int \frac{6 \varepsilon_{n, p}\left(a_{n}+a_{p}\right)^{12}}{|\vec{x}-\vec{y}|^{12}} c_{p}(\vec{y}) d \vec{y} .
$$

Similarly, we have the repulsive term for $c_{p}$.

$$
\mu_{c_{p}}^{r}=\int \frac{12 \varepsilon_{p, p}\left(a_{p}+a_{p}\right)^{12}}{|\vec{x}-\vec{y}|^{12}} c_{p}(\vec{y})+\int \frac{6 \varepsilon_{p, n}\left(a_{p}+a_{n}\right)^{12}}{|\vec{x}-\vec{y}|^{12}} c_{n}(\vec{y}) d \vec{y} .
$$

\section{REFERENCES}

[1] J.D. Jackson, Classical Electrodynamics, 3rd, Wiley, New York, 1998.

[2] C. Lee, H. Lee, Y. Hyon, C. Liu and T.C. Lin, Renormalized Poisson-Boltzmann equations: one-dimensional solutions, Nonlinearity, submitted, 2010.

[3] P.G. Cialet, The Finite Element Method for Elliptic Equations, North-Holland, Amsterdam, 1978.

[4] F. Brezzi and M. Fortin, Mixed and Hybrid Finite Element Methods, Springer-Verlag, New York, 1991.

[5] J. Zhang, X. Gong, C. Liu, W. Wen and P. Sheng, Electrorheological fluid dynamics, PRL, 101, 194503-1-4, 2008. 
[6] R. Ryham, An Energetic Variational Approach To Mathematical Modeling Of Charged Fluids: Charge Phases, Simulation And Well Posedness, Thesis, Pennsylvania State University, 2006.

[7] J. Xu and L. Zikatanov, A monotone finite element scheme for convection-diffusion equations, Math. Comput., 68(228), 1429-1446, 1999.

[8] F. Brezzi, L.D. Marini, S. Micheletti, P. Pietra, R. Sacco and S. Wang, Finite element and finite volume discretizations of drift-diffusion type fluid models for semiconductors, Centre National de la Recherche Scientifique Paris, France, Technical Report, 2002-1302, 2002.

[9] R. Ryham, C. Liu and L. Zikatanov, An mathematical models for the deformation of electrolyte droplets, Discrete Contin. Dyn. Syst. Ser., B 8(3), 649-661, 2007.

[10] R. Ryham, C. Liu and Z.Q. Wang, On electro-kinetic fluids: one dimensional configurations, Discrete Contin. Dyn. Syst. Ser., B 6(2), 357-371, 2006.

[11] J. Zhang, X. Gong, C. Liu, W. Wen and P. Sheng, Electrorheological fluid dynamics, Phys. Review Lett., 101(19), 194503-1-4, 2008.

[12] Y. Hyon, D.Y. Kwak and C. Liu, Energetic variational approach in complex fluids: maximum dissipation principle, DCDS-A, 24(4), 1291-1304, 2010.

[13] P.A. Markowich, The Stationary Seminconductor Device Equations, Springer-Verlag, Vienna, 1986.

[14] J.H. Park and J.W. Jerome, Qualitative properties of steady-state Poisson-Nernst-Planck systems: mathematical study, SIAM J. Appl. Math., 57(3), 609-630, 1997.

[15] B. Eisenberg, Ionic channels in biological membranes: natural nanotubes, Acc. Chem. Res., 31, 117-123, 1998.

[16] W. Nonner, D.P. Chen and B. Eisenberg, Progress and prospects in permeation, J. Gen. Physiol., 113, 773-782, 1999.

[17] D. Boda, W. Nonner, M. Valiskó , D. Henderson, B. Eisenberg and D. Gillespie, Steric selectivity in Na channels arising from protein polarization and mobile side chains, Biophys. J., 93, 1960-1980, 2007.

[18] B. Eisenberg, Y. Hyon and C. Liu, Energy variational analysis EnVarA of ions in water and channels: field theory for primitive models of complex ionic fluids, J. Chem. Phys., 133, 104104-1-23, 2010.

[19] P. Graf, M.G. Kurikova, R.D. Coalson and A. Nitzan, Comparison of dynamic lattice monte carlo simulations and the dielectric selt-energy Poisson-Nernst-Planck continuum theory for model ion channels, J. Phys. Chem. B, 108, 2006-2015, 2004.

[20] R.D. Coalson and M.G. Kurikova, Poisson-Nernst-Planck theory approach to the calculation of current through biological ion channels, IEEE Transaction on NanoBioscience, 4, 81-93, 2005.

[21] M.S. Kilic and M.Z. Bazant, Steric effects in the dynamics of electrolytes at large applied voltages. II. Modified Poisson-Nernst-Planck equations, Phys. Review, E75, 021503-1-11, 2007.

[22] A.L. Hodgkin and A.F. Huxley, A qualitative description of the membrane current and its application to conduction and excitation in nerve, J. Physiology, 117, 500-544, 1952.

[23] J.P. Keener and J. Sneyd, Mathematical Physiology, Springer-Verlag, New York, 1998.

[24] B. Hille, Ion Channels of Excitable Membranes, 3rd Edition, Sinauer Associates, Inc., 2001.

[25] D. Purves, Neuroscience, 4th Edition, Sinauer Associates, Inc., 2007.

[26] C. Koch, Biophysics of Computation, Oxford University Press, New York, 2004.

[27] Y. Mori, J.W. Jerome and C.S. Peskin, A Three-dimensional model of cellular electrical activity, Bulletin of the Institute of Mathematics Academia Sinica, 2(2), 367-390, 2007.

[28] Y. Mori, A Three-Dimensional Model of Cellular Electrical Activity, PhD thesis, New York University, 2007.

[29] J.K. Percus, Equilibrium State of a classical fluid of hard dods in an external field, J. Stat. Phys., 15, 505-511, 1976.

[30] T.K. Vanderlick, H.T. Davis and J.K. Percus, The statistical mechanics of inhomogeneous hard rod mixtures, J. Chem. Phys., 91(11), 7136-7145, 1989.

[31] J.P. Hansen and I.R. McDonald, Theory of Simple Liquids, Academic Press, London, 1986.

[32] N.F. Carnahan and K.E. Starling, Equation of state for nonattracting rigid spheres, J. Chem. Phys., 51(2), 635-636, 1969.

[33] J.K. Percus and G.J. Yevick, Analysis of classical statistical mechanics by means of collective coordinates, Phys. Rev., 110(1), 1-13, 1958.

[34] J.C. Lebowitz, Exact solution of generalized Percus-Yevick equation for a mixture of hard spheres, Phys. Rev., 133(4A), 895-899, 1964.

[35] Y. Rosenfeld, Free-energy model for the inhomogeneous hard-sphere fluid mixture and densityfunctional theory of freezing, Phys. Rev. Lett., 63(9), 980-983, 1989. 
[36] Y. Rosenfeld, Free-energy model for the inhomogeneous hard-sphere fluid in D dimensions: structure factors for the hard-disk $(D=2)$ mixtures in simple explicit form, Phys. Rev. A, 42(10), 5978-5989, 1990.

[37] H. Hansen-Goos and R. Roth, A new generalization of the Carnahan-Starling equation of state to additive mixtures of hard spheres, J. Chem. Phys., 124, 154506-1-8, 2006.

[38] R. Roth, Fundamental measure theory for hard-sphere mixtures: a review, J. Phys., Condens. Matter, 22(6), 063102-1-18, 2010.

[39] D. Gillespie, M. Valiskó and D. Boda, Density functional theory of the electrical double layer: the RFD functional, J. Phys., Condens. Matter, 17, 6609-6626, 2005.

[40] D. Gillespie, Energetics of divalent selectivity in a calcium channel: the ryanodine receptor case study, Biophys. J., 94(4), 1169-1184, 2008.

[41] J. Barthel, H. Krienke and W. Kunz, Physical Chemistry of Electrolyte Solutions: Modern Aspects, Springer-Verlag, New York, 1998.

[42] W.R. Fawcett, Liquids, Solutions, and Interfaces: From Classical Macroscopic Descriptions to Modern Microscopic Details, Oxford University Press, New York, 2004.

[43] L.L. Lee, Molecular Thermodynamics of Electrolyte Solutions, World Scientific, Singapore, 2008.

[44] K.S. Pitzer, Thermodynamics, McGraw Hill, New York, 1995.

[45] M.Z. Bazant, K. Thornton and A. Ajdari, Diffuse-charge dynamics in electrochemical systems, Physical Review E, 70, 021506-1-24, 2004.

[46] IBM Research, Damocles Web Site: http://www.research.ibm.com/DAMOCLES/home.html., 2007.

[47] B. Eisenberg, Living Transistors: a physicist's view of ion channels. Version 2., 1-24, 2008. http://arxiv.org/abs/q-bio/0506016v2

[48] R. Eisenberg and D. Chen, Poisson-Nernst-Planck (PNP) theory of an open ionic channel, Biophysical J., 64, A22, 1993.

[49] J.W. Jerome, Analysis of Charge Transport. Mathematical Theory and Approximation of Semiconductor Models, Springer-Verlag, New York, 1995.

[50] P.A. Markowich, C.A. Ringhofer and C. Schmeiser, Semiconductor Equations, Springer-Verlag, New York, 1990

[51] S. Selberherr, Analysis and Simulation of Semiconductor Devices, Springer-Verlag, New York, 1984.

[52] Y. Rosenfeld, Geometrically based density-functional theory for confined fluids of asymmetric ("complex") molecules, Chemical Applications of Density-Functional Theory. B.B. Laird, R.B. Ross and T. Ziegler. Washington, D.C., American Chemical Society, 629, 198-212, 1966.

[53] Y. Rosenfeld and L. Blum, Statistical mechanics of charged objects: General method and application to simple systems, J. Chem. Phys., 85, 1556-1566, 1986.

[54] Y. Rosenfeld, M. Schmidt, H.L. Wen and P. Tarazona, Fundamental-measure free-energy density functional for hard spheres: Dimensional crossover and freezing, Physical Review E, 55, 4245-4263, 1997.

[55] R. Evans, Density Functionals in the Theory of Nonuniform Fluids, Fundamentals of Inhomogeneous Fluids. D. Henderson. Marcel Dekker: 606, New York, 1992.

[56] D. Gillespie, W. Nonner and R.S. Eisenberg, Coupling Poisson-Nernst-Planck and Density Functional theory to calculate ion flux, J. Phys., Condens. Matter, 14, 12129-12145, 2002.

[57] D. Henderson, Fundamentals of Inhomogeneous Fluids, Marcel Dekker, New York, 1992.

[58] R.M. Accascina and R.M. Fuoss, Electrolytic Conductance, Interscience, New York, 1959.

[59] R.M. Fuoss and L. Onsager, Conductance of strong electrolytes at finite dilutions, Proc. Natl. Acad. Sci., 41(5), 274-283, 1955.

[60] J.C. Justice, Conductance of electrolyte solutions, Comprehensive Treatise of Electrochemistry, Thermondynbamic and Transport Properties of Aqueous and Molten Electrolytes, B.E. Conway, J.O.M. Bockris and E. Yaeger. Plenum, New York, 5, 223-338, 1983.

[61] V. Barcilon, D.P. Chen, R.S. Eisenberg and J.W. Jerome, Qualitative properties of steady-state Poisson-Nernst-Planck systems: perturbation and simulation study, SIAM J. Appl. Math., 57(3), 631-648, 1997.

[62] A. Singer and J. Norbury, A Poisson-Nernst-Planck model for biological ion channels - An asymptotic analysis in a Three-Dimensional narrow funnel, SIAM J. Appl. Math., 70(3), 949-968, 2009.

[63] B. Eisenberg and W. Liu, Poisson-Nernst-Planck systems for ion channels with permanent charges, SIAM J. Math. Anal., 38(6), 1932-1966, 2007.

[64] I. Rubinstein, Electro-Diffusion of Ions (Studies in Applied and Numerical Mathematics), SIAM, Philadelphia, PA, 1990. 
[65] W. Kunz, Specific Ion Effects, World Scientific Publishing, Singapor, 2009.

[66] P.A. Martin, Sum Rules in Charged Fluids, Reviews of Modern Physics, 60, 1076-1127, 1988.

[67] A.L. Hodgkin, The ionic basis of electrical activity in nerve and muscle, Biological Reviews, 26, 339-409, 1951.

[68] A.L. Hodgkin and A.F. Huxley, Movement of radioactive potassium and membrane current in a giant axon, J. Physiol., 121, 403-414, 1953.

[69] A.L. Hodgkin and R.D. Keynes, The potassium permeability of a giant nerve fibre, J. Physiol., 128, 61-88, 1955.

[70] P.F. Baker, M.P. Blaustein, A.L. Hodgkin and R.A. Steinhardt, The influence of calcium on sodium efflux in squid axons, J. Physiol., 200, 431-458, 1969.

[71] M.P. Blaustein and W.J. Lederer, Sodium/Calcium exchange: its physiological implications, Physiological Reviews, 79, 763-854, 1999.

[72] H.T. Davis, Statistical Mechanics of Phases, Interfaces, and Thin Films, Wiley-VCH, New York, 1996.

[73] D. Kinderlehrer and N.J. Walkington, Approximation of parabolic equations using the Wasserstein metric, Math. Modeling and Numer. Anal., 33(4), 837-852, 1999. 